\title{
Estudo correlacional entre suporte organizacional, danos relacionados ao trabalho e qualidade de vida em trabalhadores brasileiros
}

Correlational study between organizational support, work-related harm, and quality of life in Brazilian workers

Estudio correlacional entre apoyo organizacional, daño laboral, y calidad de vida en trabajadores brasileños

Recebido: 20/06/2021 | Revisado: 25/06/2021 | Aceito: 01/07/2021 | Publicado: 14/07/2021

\author{
Heitor César Costa Oliveira \\ ORCID: https://orcid.org/0000-0003-4778-6613 \\ Universidade Potiguar, Brasil \\ E-mail: cartoriosbrasil@gmail.com \\ Natália Holanda Mendes Maia de Paula \\ ORCID: https://orcid.org/0000-0002-1421-905X \\ Laureate International Universities, Brasil \\ E-mail: nataliaholandammp@gmail.com \\ Lais Karla da Silva Barreto \\ ORCID: https://orcid.org/0000-0002-5267-5355 \\ Universidade Potiguar, Brasil \\ E-mail: laisbarreto@unp.br \\ Nilton Soares Formiga \\ ORCID: https://orcid.org/0000-0003-4907-9736 \\ Universidade Potiguar, Brasil \\ E-mail: nsformiga@yahoo.com \\ Alda Karoline Lima da Silva \\ ORCID: https://orcid.org/0000-0001-7548-8897 \\ Universidade Potiguar, Brasil \\ E-mail: aldalima@unp.br
}

\begin{abstract}
Resumo
A presente investigação tem por objetivo realizar um estudo correlacional entre os construtos de suporte organizacional (SO), danos relacionados ao trabalho (DRT) e qualidade de vida (QV) no contexto da saúde do empregado brasileiro nas áreas da psicologia e de fisioterapia. Participaram da pesquisa 228 trabalhadores de organização pública e/ou privada, mediante respostas à Escala de Percepção de Suporte Organizacional (EPSO), Escala de Avaliação dos Danos Relacionados ao Trabalho (ADRT) e Escala de Qualidade de Vida (Medical Outcomes Study36. SF-36), além de informarem dados sociodemográficos por meio da solução eletrônica google forms. A análise de dados foi realizada usando o programa estatístico SPSSWIN - versão 24.0. Os resultados revelaram que o SO influenciou de forma negativa e significativa, apenas a variável DRT e suas respectivas dimensões (físico, psicológico, social). Em relação à QV, os achados dos domínios específicos apresentaram-se baixos e não significativos. A originalidade do artigo está na abordagem de uma lacuna científica, uma vez que outros estudos não exploram a importância da existência e/ou ausência de percepção do SO no público pesquisado e tem como implicações gerenciais a indicação da necessidade de programas de intervenção multiprofissionais, com o objetivo de prevenção, diagnóstico e promoção da saúde dos trabalhadores.
\end{abstract}

Palavras-chave: Suporte organizacional; Danos relacionados ao trabalho; Qualidade de vida.

\section{Abstract}

The present investigation aims to carry out a correlational study between the constructs of organizational support (OS), work-related harm (DRT) and quality of life (QL) in the context of Brazilian employee health in the areas of psychology and physiotherapy. 228 workers from public and/or private organizations participated in the research, through answers to the Organizational Support Perception Scale (EPSO), Work-Related Injury Assessment Scale (ADRT) and Quality of Life Scale (Medical Outcomes Study36. SF- 36), in addition to informing sociodemographic data through the google forms electronic solution. Data analysis was performed using the statistical program SPSSWIN - version 24.0. The results revealed that the OS influences in a negative and significant way, only the DRT variable and its respective dimensions (physical, psychological, social). Regarding QoL, the findings of specific domains were low and not significant. The originality of the article lies in the approach to a scientific gap, since other studies do not explore the importance of the existence and/or absence of perception of the OS in the 
researched public and has as managerial implications the indication of the need for multidisciplinary intervention programs, with the objective of prevention, diagnosis and promotion of workers' health.

Keywords: Organizational support; Work-related harm; Quality of life.

\section{Resumen}

La presente investigación tiene como objetivo realizar un estudio correlacional entre los constructos de apoyo organizacional (AO), daño relacionado con el trabajo (DRT) y calidad de vida (CV) en el contexto de la salud de los empleados brasileños en las áreas de psicología y fisioterapia. 228 trabajadores de organizaciones públicas y / o privadas participaron en la investigación, mediante de respuestas a la Escala de Percepción de Apoyo Organizacional (EPSO), Escala de Evaluación de Daños Relacionados con el Trabajo (ADRT) y Escala de Calidad de Vida (Estudio de Resultados Médicos36. SF-36), además de informar datos sociodemográficos mediante de la solución electrónica de formularios de google. El análisis de los datos se realizó mediante el programa estadístico SPSSWIN - versión 24.0. Los resultados revelaron que la SG influyó de manera negativa y significativa, solo en la variable DRT y sus respectivas dimensiones (física, psicológica, social). Con respecto a la CV, los hallazgos de dominios específicos fueron bajos y no significativos. La originalidad del artículo radica en el abordaje de un vacío científico, ya que otros estudios no exploran la importancia de la existencia y / o ausencia de percepción de la AO en el público investigado y tiene como implicaciones gerenciales la indicación de la necesidad de una multidisciplinariedad programas de intervención, con el objetivo de prevención, diagnóstico y promoción de la salud de los trabajadores.

Palabras clave: Apoyo organizacional; Daños relacionados con el trabajo; Calidad de vida.

\section{Introdução}

Nos últimos anos o conceito de trabalho vem sendo ressignificado para o homem, bem como a relação que ocorre nesse binômio homem-trabalho. Isso advém como resultado de mudanças do capitalismo na pós-modernidade e pelo ritmo de desenvolvimento mercadológico mundial, que impõe um maior tempo de dedicação do trabalhador ao exercício laboral, seja presencialmente ou a distância. Desse modo, destaca-se a importância da atividade ocupacional que, por um lado proporciona ao sujeito um modo de dar sentido à vida, pela representatividade interna e externa que tem no eu (Silva, Carabalho, Jucá, \& Machado, 2015) mas, por outro lado, pode comprometer a qualidade de vida e causar danos relacionados à saúde.

De fato, o trabalho é uma das fontes de dignidade para pessoa humana, fazendo sentir-se completo e ocupando, na maioria das vezes, a centralidade da vida do trabalhador. Em um ambiente de rápidas mudanças essa importância pode impactar de maneira positiva e/ou negativa em outros campos da vida pessoal (Campos, 2008). Para Mendes (2010), no Brasil, existe em curso uma importante reconfiguração nas áreas políticas, sociais, econômicas, demográficas e epidemiológicas resultantes, entre outros fatores, de novos arranjos produtivos que alteram os processos de trabalho rapidamente em busca de maior eficiência, causando constantes mudanças e instabilidade recorrente no ambiente de trabalho.

Essa contínua e célere reengenharia produtiva, em conjunto com o distanciamento das relações sociais no ambiente de trabalho, vem causando reflexos físicos, psicológicos e sociais na vida e na subjetividade do trabalhador. A crescente fragilização das relações de trabalho, o distanciamento laboral e social e as excessivas coações psicológicas por resultados, sem um reconhecido suporte prestado pelas organizações, contribuem para um estresse acima do normal no trabalhador. Logo, nesse ponto, os novos modus operandi das organizações têm afetado a qualidade de vida dos funcionários e servidores, comprometendo a saúde de uma forma geral (Mendes, 2010).

Ademais, a tensão imposta por novos arranjos laborais resultando em acumulação de funções, monitoramento constante da produção, necessidade de atenção e concentração máximas do trabalhador para realizar as atividades com eficácia e com "erro zero" são fatores que interferem no aumento da frequência de distúrbios osteomusculares relacionados ao trabalho (DORT) que, neste artigo, doravante será tratado como danos relacionados ao trabalho, com a sigla (DRT). Por conseguinte, esses aspectos comprometem o bem-estar, o engajamento com o trabalho e o desempenho do trabalhador, tendo um efeito contrário ao que deseja os gestores de equipes (Brasil, 2003; Ceribeli \& Barbosa, 2019). 
Para revelar algumas dessas consequências na organização, os indicadores dos danos causados à saúde dos trabalhadores mostram o impacto em resultados operacionais e gastos financeiros, registrados como contingências laborais que afetam o planejamento estratégico de toda instituição revelado pelo não alcance de objetivos e metas. Na visão microlaboral, ocorre o desgaste para os trabalhadores que permanecem produzindo para suprir a produção de quem está afastado ou trabalhando com capacidade de produção reduzida. Assim, o erro de dimensionamento operacional é tratado como uma consequência da fragilidade do trabalhador e não como uma falha da administração (Paschoal \& Tamayo, 2004).

Isso demonstra, dentre outras coisas, a apatia da gestão na efetividade do SO, o que faz avançar o processo de desencadeamento da migração de saúde para doença, não só do próprio indivíduo adoecido, mas de toda a cadeia de valor da instituição. Mas, de fato, o impacto está na relação do indivíduo com o trabalho, na imagem desse trabalhador junto ao seu chefe imediato e à equipe que pertence a toda organização; assim, o adoecimemto muitas vezes resulta em perda de oportunidade atual e futura de uma promoção na estrutura hierárquica e remuneratória da organização, em razão da demonstração de "fragilidade" à doença (Primo, Pinheiro, \& Sakurai, 2007).

Dessa forma, os trabalhadores quando acometidos por algum tipo de doença, passam a ser estigmatizados no ambiente de trabalho, sofrem discriminações, constrangimentos, cobranças de produtividade, humilhações e pressões dos seus superiores (Silva, Oliveira, \& Zambroni-de-Souza, 2011). Em alguns casos, os trabalhadores até percebem que estão desencadeando estresse e/ou doenças psicológicas, mas como forma de autoafirmação frente ao grupo ou a seu superior sentem orgulho desse estado, como reconhecimento do próprio esforço e virilidade, aceitando então como natural o adoecimento pelo esforço laborativo em algum momento da vida ou até escondem o que estão sentindo para se sentirem seguros.

Dal Ben et al (2004) afirmam que a atividade laboral é uma forma de ambientação social que apresenta influências psíquicas e físicas no trabalhador. O esperado é que o cumprimento da atividade laboral deveria ser causador de desenvolvimento humano, realizações, conquistas pessoais, profissionais e sociais na coletividade. Todavia, dependendo do ambiente em que o trabalho ocorre, observam-se sentimentos de descontentamento, sofrimento psíquico e físico, enfermidades, adoecimento, danos e até mesmo morte (Dale \& Dias, 2018). Na compreensão de Dejours, Dessors e Desriaux (1993), o indivíduo enfrenta no ambiente de trabalho, atividades desafiadoras e gratificantes, mas também conflituosas e desgastantes.

A primeira situação gera interesse, satisfação e desenvolvimento intelectual; a segunda desencadeia fadiga, desgaste e danos em diversas perspectivas do indivíduo. Por isso, tanto por um lado como pelo outro, a atividade laboral tende a ter reflexos na vida e no comportamento do indivíduo, seja de forma positiva e equilibrada ou negativa e desequilibrada. A esse respeito Ferreira e Mendonça (2012) dizem que o processo de saúde-doença é o objeto de estudo das relações sociais com as condições de vida e de trabalho dentro das dimensões da esfera humana identificadas como física, psíquica e social, nas quais cada uma contribui de forma significativa, tanto para a promoção de saúde ou quanto para o desencadeamento de doenças.

Essas dimensões são propulsoras para questionamentos de medidas de avaliação e cuidados com os trabalhadores inseridos no ambiente organizacional. Assim, salientar questões relativas ao apoio, reciprocidade e segurança laboral, só evidencia a importância do construto do suporte organizacional (SO). Esse construto é caracterizado pelas crenças globais que os trabalhadores manifestam sobre a organização, incluindo a valorização que a organização dá às suas contribuições e ao seu bem-estar (Byrne \& Hochwarter, 2008).

A partir dessa premissa, percebe-se a importância da existência de trocas e benefícios mútuos, em que a organização possui obrigações legais, morais e financeiras com seus empregados, por outro lado espera-se um bom desempenho, comprometimento e resultados dos trabalhos (Chong, White, \& Prybutok, 2001; Formiga \& Souza, 2014). Desse modo, podese afirmar que o SO é o modo em que o trabalhador percebe que a organização na qual ele faz parte valida as suas necessidades e desejos. Após essa reflexão, vislumbra-se o que se entende por Teoria de Suporte Organizacional (TSO), na qual o 
trabalhador se comporta dentro e para a organização, em um claro interesse direto de reciprocidade (Formiga \& Souza, 2014; Fonseca, 2019).

Nesse passo, a ideia geral do SO baseia-se então na mutualidade entre organização/trabalhador. Essa mutualidade é acionada quando o trabalhador apresenta um desempenho desejável na medida em que a organização o trata bem; ou, pelo outro lado, que o trabalhador não se sente na obrigação de contribuir com a organização, quando percebe que a instituição não se importa (mais) com ele (Dabos \& Rousseau, 2004). Segundo Siqueira (2005) essa reciprocidade nas relações trabalhadororganização é regida por um princípio moral e até físico (ação $\rightarrow$ reação) que define como esperado, o ato de retribuir por uma ação recebida, criando um ambiente de auxílio mútuo.

É a prática do trabalho, independente de qual seja desempenhada, que auxilia na construção de identidade do indivíduo, na sensação de realização, na ocupação de espaço social com interferência na qualidade de vida do trabalhador, como também nas pessoas que o cercam. Para Mendes e Morrone (2002), a afetação subjetiva do trabalho acontece com três pilares: o primeiro, a subjetividade dele (envolve a história de vida e a estrutura da personalidade do indivíduo); o segundo, saber fazer (engloba a inteligência prática, habilidades, na qual ajuda o trabalhador a executar às funções prescritas por meio de sua imaginação) e o terceiro, o coletivo do trabalho (estruturado com base nas normas e valores que instituem as relações sociais no âmbito organizacional, elaborada por meio de argumentações éticas).

Outrossim, o SO engloba crenças, valores, trocas e benefícios mútuos entre a organização e o trabalhador, onde a instituição empregatícia tem o dever de proporcionar aos seus colaboradores condições apropriadas para a realização das atividades laborais (Byrne \& Hochwarter, 2008; Formiga \& Souza, 2014). Formiga, Sousa e Freire (2018) em um estudo cujo objetivo foi avaliar a relação entres as variáveis do SO e o capital psicológico no trabalho (CPT), observaram uma relação positiva entre SO e a soma total do CPT, assim como, as dimensões que são geradas desse construto (otimismo, resiliência, autoeficácia e esperança). Diante disso, pode-se perceber a importância de um SO que integre a saúde emocional e profissional na organização, o que permitirá gerar melhor produtividade e desempenho na rotina laboral.

No que diz respeito ao dano psicológico, ele é configurado como patologia e definido como uma disfunção, um transtorno ou desenvolvimento psicorgânico ou psicogênico que atinge a esfera afetiva, limita a capacidade humana no âmbito individual, familiar, social, laboral e de lazer, interferindo no equilíbrio de todo o corpo. O dano psíquico é caracterizado pela presença dos seguintes fatores: a) agente que causa o dano, b) o sujeito que sofre o dano, e c) o nexo causal (Guedes, 2008).

No caso do nexo causal, refere-se às causas ou condutas relevantes que atuam como antecedentes que produzem um dano capaz de gerar o desequilíbrio do trabalhador. Logo, pode-se dizer que hipoteticamente a retirada da ação predominante (não todas as ações) ou na ausência do ato omissivo do agente causador, o dano não ocorreria ou não a fetaria o trabalhador. Tudo isso, examinando o caso concreto e ponderando o conjunto probatório. Nesse raciocínio, embora muitos definam o estresse, com base em um senso comum, como meros aborrecimentos do cotidiano laboral, outra perspectiva apresenta o estresse como um desgaste do organismo na busca de manter a homeostase interna, o que pode motivar desequilíbrios psíquicos e fisiológicos no indivíduo ao enfrentar episódios que o irritam, amedrontam e o excitam para além do normal (Pafaro \& De Martino, 2004).

Mesmo assim, o fato de o sofrimento psíquico não ser de caráter tangível e ser menos perceptível do que os danos físicos, dificulta a formação do chamado 'nexo causal' entre trabalho e adoecimento (Borsoi, 2007). Não obstante a isso, apresenta-se como importantes agentes causadores de sofrimentos e alterações na saúde psíquica em ambientes laboral: o assédio, a excessiva divisão de tarefas, o rigoroso controle sobre o método, a exigência sem limites de ritmo cada vez mais acelerado no cumprimento das atividades, o excesso de jornada de trabalho para cumprir objetivos e metas, políticas 
exploradoras de gestão na área de recursos humanos, a hierarquia impositiva, os multicanais de recepção e resposta de comunicação com o trabalhador e, principalmente, a falta de planejamento da gestão (Jacques \& Amazarray, 2006).

Em consequência disso, Guimarães e Grubits (2004) afirmam que é comum os trabalhadores acometidos por distúrbios funcionais apresentarem sintomas como depressão, desânimo, baixa autoestima, irritabilidade, pensamentos negativos e distúrbios do sono. Para Cruz e Maciel (2005), o diagnóstico de dano psicológico deve ser observado e compreendido com alicerce em fundamentos que facilitem a análise do caso concreto e a prevenção da prática danosa à saúde do trabalhador, de forma a coibir comportamentos reiterados que se acumulam ao longo do tempo e, muitas vezes, passam a ser considerados como normais em um ambiente produtivo.

Nesse sentido, os autores apresentam cinco linhas de análise: (a) instituir vínculo entre a condição vigente dos envolvidos, incluindo suas qualidades, habilidades e aptidões que foram modificadas e o episódio danoso referido; (b) reconhecer a existência de adversidades antecedentes; (c) realizar um estudo de diagnóstico longitudinal, no qual é possível destacar a história precedente; (d) especificar o dano avaliado fundamentado na Classificação Internacional das Doenças (CID), mediante linguagem clara e objetiva e, por fim, (e) institucionalizar o procedimento rotineiro e célere de diagnósticos de transtornos psicológicos, indicando quais as probabilidades de se tornarem crônicos.

Para a Organização Mundial de Saúde (WHOQOL-OMS, 1995), "a percepção do indivíduo de sua posição na vida, no contexto de cultura e sistema de valores em que vive e em relação às suas expectativas, seus padrões e suas preocupações" define a qualidade de vida (QV). Abordando as dimensões da QV, Ogata e Simurro (2019) afirmam que o conceito de QV envolve as dimensões física, emocional, social, ocupacional, intelectual, espiritual e ambiental, além de subjetividade e não devem ser observadas sob um aspecto exclusivo, mas de forma que sejam associadas devido à multidimensionalidade do problema.

Especificamente sobre a qualidade de vida no trabalho (QVT), a Organização Mundial de Saúde (OMS) sugere que pode ser entendida como "percepção dos indivíduos de que suas necessidades estão sendo satisfeitas ou, ainda, que lhes estão sendo negadas oportunidades de alcançar a felicidade e a autorrealização, com independência de seu estado de saúde físico ou das condições sociais e econômicas", não levando em consideração apenas a saúde física e mental (OMS, 1998; cf. Pereira, Formiga, \& Estevam, 2019).

Em orientação mais holística Aquino e Fernandes (2013), dizem que a QV(T) ocorre quando se olha a organização e os colaboradores como um todo, promovendo o bem-estar e a segurança no ambiente de trabalho com finalidade de garantir a satisfação na vida profissional, familiar e pessoal conjugada à produtividade dos trabalhadores. Em que pese ser essa equação uma tarefa complexa, cabe ao gestor equilibrar todos esses fatores, pois a satisfação dos funcionários proporciona à organização benefícios como redução do absenteísmo, melhor desenvolvimento no fluxo de comunicação, fortalecimento do sentimento de pertencimento, melhora da afetividade organização-trabalhador e reforça a imagem da organização perante a sociedade, com reflexo inclusive para outros novos trabalhadores desejarem fazer parte da instituição.

Outrossim, os programas de intervenções dirigidos à $\mathrm{QV}(\mathrm{T})$, como promoção da capacidade do trabalho e prevenção de doenças têm efeitos benéficos e aumentam a satisfação e o bem-estar dos colaboradores dentro da organização do trabalho, diminuindo os níveis de estresse. Dessa maneira, a organização que apoia, auxilia ou oferece assistência aos seus funcionários pode alavancar uma maior produtividade e engajamento dos trabalhadores justamente por essa situação demonstrar um SO ao trabalhador (Borges, 2017). Logo, ao considerar as reflexões aqui apresentadas, é possível destacar que o SO pode influenciar positivamente a QV e negativamente aos DRT.

Assim, ante ao exposto, considerando as reflexões sobreditas, faz-se necessário realizar um estudo correlacional entre os construtos de suporte organizacional (SO), danos relacionados ao trabalho (DRT) e qualidade de vida (QV) nas áreas da psicologia e de fisioterapia a fim de medir a existência ou não de correlações entre eles e possibilitar aos gestores, 
investimentos com maior eficiência e impacto na prevenção, diagnóstico e promoção da saúde do trabalhador brasileiro.

\section{Metodologia}

\section{Amostra}

Abordagem dessa investigação é descritiva, exploratória e correlacional com perspectiva quantitativa focada em trabalhadores de organizações públicas e privadas. A amostra foi do tipo não probabilística e intencional com técnica bola de neve e com o 'n' amostral avaliado por meio do pacote estatístico GPower 3.1 (Faul, Erdfelder, Lang, \& Buchner, 2007).

Para a coleta de dados estabeleceu-se uma probabilidade de $95 \%(\mathrm{p}<0,05)$, magnitude do efeito amostral $(\mathrm{r} \geq 0,50)$ e um padrão de poder hipotético $(\pi \geq 0,80)$. Nesses padrões trabalhou-se com uma amostra de 228 trabalhadores e indicadores estatísticos $(\mathrm{t} \geq 1,98 ; \pi=0,94 ; \mathrm{p}<0,05)$ que garantiram a qualidade da amostra com os seguintes itens sociodemográficos: idade, distribuição entre os municípios brasileiros Natal/RN e João Pessoa/PB, carga de trabalho (número de expedientes e acúmulo de função), quantidade de vínculo empregatício, tempo de serviço, sexo, estado civil, nível de instrução, especialização profissional e natureza jurídica da organização, considerada nesta investigação como pública ou privada.

\section{Instrumentos}

Os sujeitos responderam a um questionário integrado com a caracterização sociodemográfica e três escalas, quais sejam: percepção de suporte organizacional (EPSO), qualidade de vida (Medical Outcomes Study36. SF-36) e avaliação dos danos relacionados ao trabalho (EADRT).

No que diz respeito à escala de percepção de suporte organizacional (EPSO), trata-se de um instrumento desenvolvido por Eisenberger et al (1986) adaptado e validado para o contexto brasileiro por Siqueira (1995). Ele é composto por 9 itens, por exemplo: $\mathrm{SO} 1$ = Esta empresa ignoraria qualquer reclamação de minha parte]; [SO2 = Esta empresa não considera meus interesses quando toma decisões que me afetam]; [SO3 = É possível obter ajuda desta empresa quando tenho um problema, etc.

A escala avalia o quanto as pessoas que trabalham nas organizações públicas e/ou privadas percebem que a empresa em uma preocupação com o bem-estar do trabalhador, devendo o participante indicar a sua resposta marcando no item numa escala de sete pontos que variava de $1=$ discordo totalmente a 7 = concordo totalmente o quanto estaria de acordo. Essa medida foi validada por Siqueira (1995) e corroborada por Formiga, Fleury e Souza (2014) quanto à organização fatorial e qualidades psicométricas.

No que se refere à escala de qualidade de vida (Medical Outcomes Study36. SF-36), trata-se de um instrumento que tem por objetivo mensurar a qualidade de vida. Ele apresentou consistência interna satisfatória na pesquisa, o que resultou em um recurso de fácil administração. Essa escala é constituída por um questionário multidimensional formada por trinta e seis itens, distribuído em oito domínios: capacidade funcional, aspectos físicos, dor, estado geral de saúde, vitalidade, aspectos sociais, aspectos emocionais, saúde mental e avaliação comparativa do estado geral de saúde atual e de um ano atrás.

Por fim, foi utilizada a escala de avaliação de danos relacionados ao trabalho (EADRT) foi desenvolvida por Mendes (2007) tem como objetivo avaliar os danos relacionados ao trabalho (DRT), a qual, destina-se a revelar as situações graves relacionadas à saúde. É composta por vinte e nove itens, dentre eles verifica-se: dores no corpo, dor de cabeça, dificuldades com os amigos, vontade de ficar sozinho, irritação com tudo, dúvida sobre a capacidade de fazer as tarefas. Os componentes dessa escala foram agrupados em dois sumários, o físico e o mental, distribuídos ainda em 3 (três) fatores [danos físicos, danos psicológicos e danos sociais]. No questionário, o participante deverá indicar a sua resposta numa escala do tipo Likert de 07 pontos (variando da seguinte forma: $0=$ nenhuma vez; $1=$ uma vez; $2=$ duas vezes; $3=$ três vezes; $4=$ quatro vezes; $5=$ cinco vezes e $6=$ seis ou mais vezes). 


\section{Técnica de coleta de dados, procedimentos de pesquisa e análise estatística}

O instrumento da pesquisa foi aplicado em trabalhadores das cidades de Natal-RN e Fortaleza-CE de forma individual, por intermédio de um formulário eletrônico hospedado no google.forms. De acordo com a disponibilidade do tempo e espaço físico dos respondentes em seus setores de trabalho ou fora dele, eles foram convidados a responder o questionário eletrônico de forma voluntária, privada e sem identificação. O formulário também foi encaminhado para o e-mail e mensageiros eletrônicos (aplicativos) dos trabalhadores. Foi informado aos participantes sobre o tratamento, anonimização dados e sigilo dados, inclusive da opção de desistência da participação a qualquer momento e eliminação dos dados.

Ademais, para aplicação do instrumento, o pesquisador ficou disponível durante todo o momento da aplicação do instrumento para responder às dúvidas por e-mail ou pelo próprio mensageiro eletrônico. Esse procedimento teve por objetivo dar suporte e esclarecer possíveis incompreensões do instrumento. Um tempo médio de 27 minutos foi suficiente à conclusão das respostas da pesquisa.

Todos os procedimentos adotados nesta pesquisa seguiram as orientações previstas na Resolução 466/2012 do CNS e na Resolução 016/2000 do Conselho Federal de Psicologia para as pesquisas com seres humanos (Conselho Nacional de Saúde [CNS], 2012; Associação Nacional de Pesquisa e Pós-Graduação em Psicologia [ANPEPP], 2000), submetido ao CONEP na Plataforma Brasil e aprovado sob o protocolo de pesquisa CAAE no 28789319.6.0000.5568. Em relação à análise dos dados, utilizou-se o pacote estatístico SPSSWIN em sua versão 24.0 para tabular os dados, realizar as análises estatísticas descritivas e os cálculos referentes à correlação de Pearson, teste $t$ de Student, alfa de Cronbach e qui-quadrado.

\section{Resultados e Discussão}

Finalizada a fase de coleta dos dados e categorizadas as respostas no programa excel, ato contínuo, foram copiadas para o pacote estatístico SPSS.24, quando foram verificados, por meio de uma inspeção visual, os valores mínimos e máximos dos respondentes nas escalas apresentadas, a fim de garantir a qualidade da amostra. Observou-se as seguintes características: a maioria foi do sexo masculino (56\%), todos acima de 18 anos, 50\% casados, 37\% solteiros, 9\% com relações estáveis e 3\% divorciados. Quanto à instrução $43 \%$ têm o curso superior e $43 \%$ têm especialização. Quanto ao setor funcional, $72 \%$ são funcionários de empresas privadas. Quanto ao tempo de serviço, eles têm em média 7,02 anos. No que tange ao vínculo 65\% têm apenas um único vínculo de trabalho. Quanto ao horário de trabalho, 78\% trabalham os dois expedientes (manhã e tarde) e quanto à carga de trabalho $61 \%$ dos respondentes afirmam que têm atividades de acumulação exercendo mais de uma função. Em relação aos fatores sócio-laborais, $72 \%$ já sentiram dores no corpo devido à quantidade de trabalho na organização. No que diz respeito às atividades físicas, 79\% afirmaram ter alguma atividade e 54\% fazem essa atividade três vezes na semana.

Quanto à qualidade da amostra, no que se refere aos dados omissos das pesquisas, observou-se que não foram maiores que 5\%. Desse modo, caso fossem encontrados no banco de dados missing ou dados duplicados, seria possível substituir pela média ou moda dos dados da amostra (Tabachnick \& Fidell, 2001). Em relação à multicolinearidade entre as variáveis, observou-se a existência de correlações (r) entre elas $\leq 0,90$, variando de 0,34 a 0,65. Na verificação dos outliers multivariados na amostra, o teste de normalidade de Kolmogorov-Smirnov $(K S)$ revelou que à amostra coletada apresentou uma normalidade $(\mathrm{KS}=0,72)$ da amostra a um $\mathrm{p}<0,38$.

Em relação ao estudo correlacional entre os construtos (SO, DRT, QV) convém destacar a condição na avaliação dessas correlações: quando existente correlações positivas, essas referem-se à capacidade de influência linear e direta entre as variáveis, contribuindo de forma positiva em relação à definição abordada no construto em geral e/ou nas especificidades das suas dimensões. No que diz respeito à correlação negativa, revela-se um relacionamento inverso entre as variáveis. Logo, os 
construtos que apresentarem essas correlações, podem ser destacados de forma inferencial. Para essas conclusões, efetuou-se o cálculo de correlação de Pearson entre as variáveis.

A partir dessas considerações, na tabela 1, pode-se observar a existência de correlações positivas, as quais se referem à capacidade de influência entre as variáveis, contribuindo de forma direta em relação à definição abordada no construto geral e/ou nas especificidades das suas dimensões. Assim, pode-se observar na tabela 1, que o SO (Suporte Organizacional) influenciou, de forma significativa e negativamente, apenas a variável DRT (Danos Relacionados aos Trabalho) e suas respectivas 03 (três) dimensões (DRTfísico, DRTpsicológico, DRTsocial), mas, não foi significativa a correlação referente à QV (Qualidade de Vida). Por outro lado, o questionário da QV apresentou escores correlacionais negativos e significativos com o DRTtotal. 
Research, Society and Development, v. 10, n. 8, e36810817380, 2021

(CC BY 4.0) | ISSN 2525-3409 | DOI: http://dx.doi.org/10.33448/rsd-v10i8.17380

Tabela 1: Correlação de Pearson (r) entre suporte organizacional, qualidade de vida e danos relacionados ao trabalho em brasileiros.

\begin{tabular}{|c|c|c|c|c|c|c|c|c|c|c|c|c|c|c|c|c|}
\hline $\begin{array}{l}\text { Variáveis/ } \\
\text { Construto }\end{array}$ & 1 & 2 & 3 & 4 & 5 & 6 & 7 & 8 & 9 & 10 & 11 & 12 & 13 & 14 & 15 & 16 \\
\hline $\begin{array}{l}\text { 1.Percepção do suporte organizacional } \\
\text { (SO) }\end{array}$ & --- & & & & & & & & & & & & & & & \\
\hline 2.SF36 (QVtotal) & $-0,07$ & --- & & & & & & & & & & & & & & \\
\hline 3.Domínio Físico (DF) & $-0,07$ & $0,90 *$ & --- & & & & & & & & & & & & & \\
\hline 4.Capacidade Funcional (CF) & $0,15^{*}$ & $0,48^{*}$ & $0,40^{*}$ & --- & & & & & & & & & & & & \\
\hline 5.Aspectos Físicos (AF) & $0,15 *$ & $0,37 *$ & $0,34 *$ & $0,37 *$ & --- & & & & & & & & & & & \\
\hline 6.DOR (Dor) & $-\overline{2} 6^{*}$ & $0,19 *$ & $0,34 *$ & $-0,42 *$ & $-0,30 *$ & --- & & & & & & & & & & \\
\hline 7.Estado Geral de Saúde (EGS) & $-\overline{14 *}$ & $0,57 *$ & $0,70 *$ & $-0,23 *$ & $-0,11$ & $0,39 *$ & --- & & & & & & & & & \\
\hline 8.Domínio Mental (DM) & $-0,10$ & $0,66^{*}$ & $0,37 *$ & 0,05 & 0,06 & $0,38 *$ & $0,26 *$ & --- & & & & & & & & \\
\hline 9.Saúde Mental (SM) & $0,16^{*}$ & $0,20 *$ & $0,37 *$ & $-0,28 *$ & $-0,23 *$ & $0,86^{*}$ & $0,36^{*}$ & $0,37 *$ & --- & & & & & & & \\
\hline 10.Aspectos Emocionais (AE) & $0,22 *$ & $0,38 *$ & $0,15^{*}$ & $0,32 *$ & $0,58 *$ & $-\overline{3}$ & $-0,12$ & $0,37 *$ & $-0,32 *$ & --- & & & & & & \\
\hline 11.Aspectos Sociais (AS) & $0,23 *$ & $0,32 *$ & $0,19 *$ & $-0,16^{*}$ & $-0,30^{*}$ & $0,48^{*}$ & $0,28 *$ & $0,64 *$ & $0,32 *$ & $-0,19 *$ & --- & & & & & \\
\hline 12.Vitalidade (VIT) & $-\overline{2} 6^{*}$ & $0,28 *$ & $0,18 *$ & $0,27 *$ & $0,20 *$ & $-\overline{0}, 38 *$ & $-0,11$ & $0,38 *$ & $-0,33 *$ & $0,32 *$ & $-0,23$ & --- & & & & \\
\hline $\begin{array}{l}\text { 13.Danos Relacionados Trabalho } \\
\text { (pontuação total) (ADRTtotal) }\end{array}$ & $-\overline{4}$ & $0,40 *$ & 0,10 & 0,12 & $-0,36^{*}$ & $0,35^{*}$ & $0,59 *$ & $0,30 *$ & 0,05 & $-0,57 *$ & $0,50 *$ & $-050^{*}$ & --- & & & \\
\hline $\begin{array}{l}\text { 14.Danos Físicos Relacionados Trabalho } \\
\text { (DRTfísico) }\end{array}$ & $0,29 *$ & 0,07 & 0,03 & $-\overline{4}+4$ & $0,36^{*}$ & $0,51 *$ & $0,24 *$ & 0,10 & $-\overline{4}$ & $-\overline{4}+4 *$ & $0,47 *$ & $-\overline{4}$ & $0,85^{*}$ & --- & & \\
\hline $\begin{array}{l}\text { 15.Danos Psicológicos Relacionados ao } \\
\text { Trabalho (ADRTpsico) }\end{array}$ & $-\overline{4}$ & $0,14 *$ & $0,20 *$ & $0, \overline{29} *$ & $-\overline{2}$ & $0,56^{*}$ & $0,32 *$ & 0,02 & $\begin{array}{c}- \\
0,55^{*}\end{array}$ & $-\overline{4}$ & $0,42 *$ & $\begin{array}{c}- \\
0,49 *\end{array}$ & $0,93 *$ & $0,66^{*}$ & --- & \\
\hline $\begin{array}{l}\text { 16.Danos Sociais Relacionados ao } \\
\text { Trabalho (DRTsoc) }\end{array}$ & $\overline{-}, 30^{*}$ & 0,04 & $0,15^{*}$ & $-\overline{19}$ & $-\overline{2} 4^{-}$ & $0,45 *$ & $0,23 *$ & 0,02 & $-\overline{-}$ & $-\overline{4}+4$ & $0,44^{*}$ & $-\overline{4}$ & $0,84 *$ & $0,56^{*}$ & $0,77 *$ & --- \\
\hline
\end{tabular}

Nota: $* \mathrm{p}<0,01$. Fonte: Dados da pesquisa (2021). 
Ainda taTbela 1, salienta-se a existência de intracorrelações entre as especificidades de cada construto e suas dimensões fatoriais. Na DRTtotal observou-se correlações positivas e significativas com as dimensões dano físico (DRTfísico), psicológico (DRTpsicológico) e social (DRTsocial), bem como, entres elas. Isto é, tanto em termos de construto quanto de mensuração do fenômeno em si nos trabalhadores, pode-se afirmar que elas medem na mesma direção conceitualmente, proposta pelos autores desse construto.

No que diz respeito à QV observou-se, entre os domínios específicos da qualidade de vida, correlações baixas (< 0,30), negativas e outras, não significativas. Essa condição poderá denotar a existência de baixas pontuações na percepção dos respondentes. Além disso, observar a influência não significativa do SO sobre a QV permitiu revelar o quanto esse construto pouco influencia os domínios da QV.

A partir dos cálculos correlacionais entre as variáveis, realizaram-se o teste $t$ de Student para verificar a variação dos escores nas variáveis de SO, DRT e a QV em função do sexo (masculino e feminino), bem como, uma ANOVA On-way, comparando a pontuação média das respostas dos sujeitos nas mesmas variáveis, respectivamente, em função do setor funcional e o tempo de serviço.

Dessa maneira, na Tabela 2 é possível o observar que todas as diferenças foram significativas e que o sexo masculino apresentou maiores escores nas variáveis-construtos do SO e da QV, enquanto o sexo feminino, revelou maiores escores nos DRT. Observadas as diferenças entre o sexo e os construtos abordados, verificou-se as variações dos escores nas variáveis em questão (SO, DRT e a QV) relativo ao setor funcional, isto é, se o respondente trabalha no setor público, privado ou em ambos. Observou-se ainda, um resultado significativo apenas para o SO e no DRT; respectivamente, o maior escore esteve para os respondentes que trabalham no setor privado e em ambos os setores (público e privado), conforme Tabela 3.

Tabela 2: Diferenças entre o Sexo no SO, DRT e QV.

\begin{tabular}{lcccccc}
\hline Construtos & Sexo & N & Média & d.p. & t & p < \\
\hline $\begin{array}{l}\text { Suporte } \\
\text { Organizacional }\end{array}$ & M & 127 & $\mathbf{3 1 , 1 9}$ & 8,62 & 3,76 & 0,01 \\
Danos Relativos & F & 95 & 27,01 & 7,58 & & 0,01 \\
ao Trabalho & M & 122 & 37,22 & 23,48 & & $-4,12$ \\
& F & 94 & $\mathbf{5 7 , 1 5}$ & 37,60 & & 0,01 \\
QV (SF36) & M & 118 & $\mathbf{7 0 , 3 6}$ & 4,30 & & 2,81 \\
& F & 91 & 68,54 & 6,42 & & \\
\hline
\end{tabular}

Nota: $\mathrm{M}=$ Masculino $; \mathrm{F}=$ Feminino $; \mathrm{QV}=$ Qualidade de Vida $; \mathrm{N}=$ amostra d.p. = Desvio Padrão. Fonte: Dados da pesquisa (2021). 
Tabela 3: Análise de Variância do SO, DRT e QV em Função do Setor Funcional.

\begin{tabular}{|c|c|c|c|c|c|c|c|}
\hline \multirow{2}{*}{$\begin{array}{c}\text { VI } \\
\text { (Construtos) }\end{array}$} & \multirow{2}{*}{$\begin{array}{c}\text { VD } \\
\text { (setor } \\
\text { funcional) }\end{array}$} & \multirow{2}{*}{ Média } & \multirow{2}{*}{ d.p. } & \multicolumn{2}{|c|}{$95 \%$ IC } & \multicolumn{2}{|c|}{ Estatística } \\
\hline & & & & Baixo & Alto & F Friedman/gl & p-valor \\
\hline \multirow{3}{*}{$\begin{array}{c}\text { Suporte } \\
\text { Organizacional } \\
(\mathrm{SO})\end{array}$} & Público & 24,41 & 8,14 & 22,05 & 26,78 & \multirow{3}{*}{$29,76 / 2$} & \multirow{3}{*}{0,01} \\
\hline & Privado & 31,69 & 7,40 & 30,55 & 32,84 & & \\
\hline & Ambos & 20,46 & 7,93 & 15,66 & 25,25 & & \\
\hline \multirow{3}{*}{$\begin{array}{c}\text { Danos Relacionados } \\
\text { ao Trabalho } \\
\text { (DRT) }\end{array}$} & Público & 49,95 & 32,34 & 40,45 & 59,45 & \multirow{3}{*}{$4,17 / 2$} & \multirow{3}{*}{0,05} \\
\hline & Privado & 43,45 & 37,66 & 37,52 & 49,39 & & \\
\hline & Ambos & 55,38 & 35,46 & 33,95 & 76,81 & & \\
\hline \multirow{3}{*}{$\begin{array}{c}\text { QV } \\
(\mathrm{SF} 36)\end{array}$} & Público & 70,00 & 4,43 & 68,69 & 71,30 & \multirow{3}{*}{$0,86 / 2$} & \multirow{3}{*}{0,42} \\
\hline & Privado & 69,31 & 5,76 & 68,39 & 70,24 & & \\
\hline & Ambos & 71,27 & 2,96 & 69,27 & 73,26 & & \\
\hline
\end{tabular}

Notas: VI = Variável Independente; VD = Variável Dependente; dp = desvio padrão; IC = Intervalo de Confiança. QV = Qualidade de Vida. Fonte: Dados da pesquisa (2021).

Em seguida avaliou-se, considerando também, o cálculo da ANOVA, em relação ao tempo de serviço; na Tabela 4 é possível observar que, apesar do resultado não ser significativo em nenhuma das variáveis, a título de curiosidade, os maiores escores estiveram distribuídos da seguinte forma: no SO esteve para os trabalhadores com menos tempo de serviço, enquanto para o DRT e a QV, os trabalhadores entre 3 e 7 anos, pontuaram escores mais alto nessas variáveis.

Tabela 4: Análise de Variância do SO, DRT e QV em Função do Tempo de Serviço.

\begin{tabular}{|c|c|c|c|c|c|c|c|}
\hline \multirow{2}{*}{$\begin{array}{c}\text { VI } \\
\text { (Construtos) }\end{array}$} & \multirow{2}{*}{$\begin{array}{c}\text { VD } \\
\text { Tempo de } \\
\text { serviço }\end{array}$} & \multirow[t]{2}{*}{ Média } & \multirow[t]{2}{*}{ d.p. } & \multicolumn{2}{|c|}{$95 \%$ IC } & \multicolumn{2}{|c|}{ Estatística } \\
\hline & & & & Baixo & Alto & $\begin{array}{l}\text { F Friedman } \\
/ \mathrm{gl}\end{array}$ & p-valor \\
\hline \multirow{3}{*}{$\begin{array}{c}\text { Suporte } \\
\text { Organizacional } \\
(\mathrm{SO})\end{array}$} & 1-2 anos & 30,28 & 8,15 & 28,33 & 32,24 & \multirow{3}{*}{$0,70 / 2$} & \multirow{3}{*}{0,52} \\
\hline & 3-7 anos & 28,70 & 8,44 & 26,81 & 30,60 & & \\
\hline & acima de 8 anos & 29,34 & 8,70 & 27,30 & 31,39 & & \\
\hline \multirow{3}{*}{$\begin{array}{c}\text { Danos } \\
\text { Relacionados } \\
\text { ao Trabalho (DRT) }\end{array}$} & 1-2 anos & 45,05 & 34,44 & 36,72 & 53,39 & \multirow{3}{*}{$1,16 / 2$} & \multirow{3}{*}{0,31} \\
\hline & 3-7 anos & $\mathbf{5 0 , 5 3}$ & 38,61 & 41,83 & 59,24 & & \\
\hline & acima de 8 anos & 41,43 & 36,15 & 32,74 & 50,12 & & \\
\hline \multirow{3}{*}{ SF36 (QV) } & $1-2$ anos & 70,09 & 5,15 & 68,79 & 71,39 & \multirow{3}{*}{$2,53 / 2$} & \multirow{3}{*}{0,08} \\
\hline & 3-7 anos & 70,40 & 4,14 & 69,46 & 71,34 & & \\
\hline & acima de 8 anos & 68,82 & 3,85 & 67,88 & 69,76 & & \\
\hline
\end{tabular}

Notas: VI = Variável Independente; VD = Variável Dependente; $\mathrm{dp}=$ desvio padrão; IC = Intervalo de Confiança. QV = Qualidade de Vida. Fonte: Dados da pesquisa (2021).

A partir dos resultados observados na presente pesquisa, é possível então refletir nas seguintes direções: inicialmente, pode-se afirmar que as medidas utilizadas nesse artigo foram consistentes, garantindo com isso a aplicação aos respondentes do estudo, bem como, para futuras pesquisas.

Os achados da pesquisa sobre as escalas de QV, SO e DRT, corroboram a proposta teórica do construto com outros autores (Laguardia, Campos, Flor e Portugal, 2015; Formiga, et al, 2018; Formiga et al, 2020; Nascimento \& Estevam, 2020), 
quanto à condição de sua avaliação ser confiável, o que permite afirmar que é possível mensurar os construtos desta investigação em trabalhadores.

Assim sendo, as escalas utilizadas neste artigo revelaram indicadores psicométricos com qualidade para a mensuração do SO e DRT. No que se refere ao instrumento de QV (SF-36), ele já vem sendo avaliado de forma bastante consistente, seja em pesquisas on-line ou físicas (com aplicação in loco), condição com amostras das mais diversas (cf. Laguardia, Campos, Flor, \& Portugal, 2016; Nascimento \& Estevam, 2020), o que tornou desnecessário realizar essa análise.

A reflexão com base nos achados desta investigação corrobora com a perspectiva de Siqueira (1995), bem como no estudo de Bizzi (2013) os quais revelaram que o SO percebido impacta, positivamente, no bem-estar e no comprometimento afetivo, condição que possibilita melhor desenvolvimento no espaço laboral e produtividade, como também influencia em outros construtos psicológicos no ambiente de trabalho, como é no caso do engajamento e espiritualidade no trabalho (cf. Formiga et al., 2019).

No que diz respeito ao estudo correlacional entre os construtos QV, SO e DRT no contexto da saúde do empregado brasileiro da área da psicologia e de fisioterapia, o SO influenciou positivamente, os aspectos mais socio-humanos (por exemplo, capacidade funcional, aspectos físicos, saúde mental e os aspectos emocionais) o que favorece uma interação social com qualidade, portanto, não só os domínios físicos, mas, os psicológicos, foram explicados por esse construto. Isto é, provavelmente, a organização que apoie (focada na valorização e bem-estar) o trabalhador terá como resultado um indivíduo mais saudável.

Os resultados associados de $\mathrm{SO}$ e dor revelaram uma relação inversa, evidenciando que o apoio da organização e o reconhecimento do limite de desempenho e capacidade funcional do trabalhador, são importantes para prevenção de interferências e prejuízos no corpo do indivíduo. Nesse ponto, Marques e Giongo (2016) corroboram essa concepção ao afirmarem em estudo com trabalhadores bancários sob sofrimento que, a precarização geral e subjetiva, as fortes pressões, o ritmo de trabalho intenso, as violências éticas e morais praticada pela gestão e gerada pela reestruturação organizacional levam ao sofrimento e desencadeiam patologias físicas e psíquicas, além de prejuízos à organização e ao trabalhador.

Um resultado não esperado foi observado na relação negativa do SO com a vitalidade e os aspectos sociais. Nesse aspecto, uma provável explicação está na possibilidade de que a percepção do SO não é suficiente para influenciar essa condição. Mas, isso foi surpreendente porque Ceribeli e Barbosa (2019) afirmam que com a baixa socialização e apoio, o trabalhador pode começar a se sentir prejudicado, afetando o humor e principalmente a vitalidade, com sinais de cansaço e esgotamento. Coelho et al (2020) complementam essa visão com um estudo em que a organização que dispõe de um ambiente de convivência, no qual os trabalhadores, independente do cargo exercido, possam construir um relacionamento mais próximo e assim compor um ambiente de trocas de experiências e conversas, promove a saúde e bem-estar do trabalhador, além de proporcionar trocas de saberes.

Considerando a relação SO e DRT foi destaque a condição de que esse construto tanto influenciou, negativamente o DRT, quanto às especificidades dos danos, isto é, físico, psíquico e social; assim, quanto melhor o SO, menores esses danos. Dessa forma, os resultados revelam que uma organização que oferece apoio aos seus funcionários terá menos danos laborais, seja um dano em sua forma geral, seja na sua especificidade de domínio. Destaque-se também que o SO, quando relacionado com as especificidades do DRTtotal, influenciou negativamente cada um dos três domínios, onde o domínio DRTpsico apresentou mais significância, seguido do domínio DRTsocial e posteriormente do domínio DRTfísico; apesar de que as diferenças entre eles foram pequenas.

No que se refere a esses domínios, um estudo relacionado aos danos à saúde dos trabalhadores de enfermagem de salas de vacinação realizado por Fonseca et al (2020), mostrou que os danos físicos foram classificados como críticos, enquanto os danos psicológicos e sociais receberam avaliação suportável. Ao considerar os resultados, acredita-se que os danos 
físicos foram mais representativos, nesse caso, devido às particularidades do trabalho na sala de vacinação, que geralmente apresentam acomodações e equipamentos com ergonomia inadequada, intenso ritmo de trabalho, movimentos repetitivos, trabalho em pé e utilização de uma postura corporal inadequada durante a administração das vacinas (Fonseca et al, 2019).

No que diz respeito à $\mathrm{QV}$, mesmo não apresentando números muito significativos, observou-se que quanto mais baixa a QV, mais alto o DRT, o que revela a importância da QV(T). Na visão de Ribeiro e Santana (2015), qualidade de vida é pautada, principalmente, pela preocupação relacionada ao bem-estar e as formas como as organizações vão buscar alternativas a fim de minimizar os danos causados pelo trabalho na saúde dos seus colaboradores. Nesse sentido, é possível reforçar que a QV desenvolvida pelos trabalhadores influencia no DRT, cabe então destacar aqui a abordagem de QV realizada por Ogata e Simurro (2019). Para os autores a QV apresenta multidimensionalidade e subjetividade, dependendo da percepção e experiências individuais de cada um. Desse modo, a afirmação de uma boa qualidade de vida é sustentada pela interação forma positiva de aspectos como: estado psicológico, relações sociais, expectativas, crenças, valores e o meio ambiente.

Ao observar os achados da QV quanto pontuação total, a relação foi positiva, condição que, provavelmente, permite pensar que os respondentes desse estudo teriam um escore baixo na $\mathrm{QV}$, mas, quanto às especificidades dos domínios desse construto, aqueles, com foco físico (aspectos físicos) de QV e os mais psicológicos (aspectos emocionais e vitalidade) influenciaram na inibição da DRT e nos danos específicos (físico, psíquico e social). É compreensível discutir esse tema porque a QV no âmbito geral pode somar todos os domínios, dificultando entender quais os domínios poderiam influenciar.

Em se tratando de diferenças entre o sexo no SO, DRT e QV os homens percebem ter mais SO e QV e as mulheres percebem ter mais DRT. Isso pode ser explicado dado que as mulheres do mundo moderno tendem a dedicarem mais tempo às atividades do lar do que os homens após o horário de trabalho, como por exemplo, tarefas domésticas, cuidar da família, criação e educação dos filhos. Assim sendo, na maioria das vezes, as mulheres sentem mais o acúmulo de sobrecarga, esgotamento físico e interferência na qualidade de vida.

Boehs, Bardabi e Silva (2019) verificaram as relações entre os aspectos referentes ao papel de trabalho exercido durante a carreira, a tomada de decisão sobre a aposentadoria e a satisfação da vida em 89 aposentados de uma empresa multinacional. Observou-se nessa pesquisa que nenhuma mulher considerou sua qualidade de vida como ótima durante a carreira de trabalho, enquanto $26,6 \%$ dos homens assinalaram consideraram.

Ainda nesse assunto, dados do relatório Perfil do Trabalho Decente no Brasil: um olhar sobre as unidades da Federação (Guimarães, 2012) revelam que as mulheres, de fato são mais sobrecarregadas do que os homens, pois trabalham cerca de 22 horas a mais por semana, sendo cinco a oito horas semanais dedicadas às atividades domésticas e as demais dedicadas à sua rotina laboral. Tal resultado sugere que as mulheres se sentem mais sobrecarregadas ao ter que lidar com trabalho e família, o que pode justificar a percepção mais direcionada ao DRT. Noutro ponto, é possível que os homens percebam ter mais SO devido à presença do discurso sexista ainda presente, relacionado ao modo de processos de instrumentalidade, aos processos de autovalorização à força, entre outros.

Outro achado é que na análise de variância entre organizações públicas e privadas, os trabalhadores das organizações privadas têm maior percepção de SO quando comparada a instituições públicas. Já os trabalhadores que atuam em ambas as organizações (públicas e privadas) reconhecem ter mais DRT e comprometimento da QV. Uma pesquisa realizada por Vey, Silva, Braz e Saccol (2018) corrobora com esse estudo onde identificou-se as queixas musculoesqueléticas e qualidade de vida em profissionais bancários de instituições públicas e privadas, onde os funcionários da rede pública relataram sentir mais dores ocasionadas pelas atividades exercidas no trabalho.

Em relação ao tempo de serviço correlacionado ao SO, trabalhadores com um a dois anos de tempo de serviço (modernos) reconheceram ter mais SO em relação aos que possuem de três a sete anos (estáveis) e acima de oito anos na 
função (experientes). Quando associado aos DRT e à QV, os trabalhadores modernos (com um a dois anos de função laboral) indicaram maiores danos relacionados ao trabalho e pior qualidade de vida.

Os resultados de uma pesquisa desenvolvida por Boehs, Bardabi e Silva (2019) apontaram que quanto melhor a percepção do trabalhador acerca da QV durante a carreira, maiores os níveis de satisfação de vida atual e o desejo de não querer se desvincular do trabalho. Isso revela que a instituição organizacional deve levar em consideração as necessidades do trabalhador, a fim de proporcionar mais qualidade de vida e, com isso, reduzir o número de afastamentos e/ou trocas de emprego.

Os trabalhadores que têm a percepção de ter mais SO são os que apresentam baixo DRT. Essa relação negativa é confirmada pelos estudos de Shantz et al. (2016), nos quais constataram que o SO dado pela organização, como incentivo ao desenvolvimento do trabalhador, implica um menor nível de esgotamento individual nas práticas de atividades laborais. $\mathrm{Na}$ mesma direção do raciocínio, destaca-se o estudo de Formiga et al (2019), pois, revelou que o SO pode ser mediado pelo engajamento na relação entre SO e espiritualidade no trabalho.

\section{Considerações Finais}

O objetivo principal do artigo foi alcançado, ou seja, foi realizado o estudo correlacional entre os construtos de suporte organizacional (SO), danos relacionados ao trabalho (DRT) e qualidade de vida (QV) no contexto da saúde do empregado brasileiro nas áreas da psicologia e de fisioterapia e apresentado os resultados. Este estudo também traz uma série de contribuições práticas. Em primeiro lugar é possível afirmar que apesar de o colaborador ver o SO como uma expectativa de futuro visando melhor qualidade de vida, ele tem de ser consciente de que a organização não é a única responsável pelos aspectos da saúde do funcionário como um todo, visto que ele tem responsabilidade de si (Formiga, Freire, Azevedo, \& Faria, 2020). Em segundo lugar, o bem-estar no trabalho, o envolvimento do trabalhador e o comprometimento afetivo da organização são componentes psicossociais que influenciam a saúde do colaborador no ambiente organizacional (Siqueira, 2014).

Em razão disso, é importante a comunicação efetiva que deixe claro aos trabalhadores as relações de trocas entre a organização e indivíduo aumentando a percepção quanto ao SO oferecido. Além disso, a organização pode intervir por meio de diagnósticos e melhorias afim de entregar aos trabalhadores uma qualidade de vida satisfatória e, consequente à essa ação, atingir os melhores resultados e desempenho por meio de da redução do absenteísmo, redução de danos relativos ao trabalho, aumento do presenteísmo devido às melhores condições de saúde do grupo de trabalhadores.

Para Dejours (2004), a prática do reconhecimento no trabalho é percebida como condição principal para a conquista da identidade no cenário social e na concepção de sentido pelo trabalho. Mas, deve-se ficar atento com a dinâmica do reconhecimento no trabalho, pois ela pode promover a conversão do sofrimento em prazer, mascarando, danos e doenças. Desse modo, percebe-se a necessidade de existir mais atenção e percepção no que concerne à saúde do trabalhador, com mais participação e comprometimento das políticas públicas e empenho das empresas com ênfase e fortalecimento da prevenção dos danos relacionados ao trabalho e no aumento da qualidade de vida do trabalhador (Vasques-Menezes, 2012).

Por fim, como sugestões para futuras investigações, percebeu-se nos resultados para percepção de SO relacionado ao tempo de serviço uma oportunidade para novas investigações, pois esperava-se que aquele trabalhador com menor tempo de serviço (modernos) se autoavaliassem com maior qualidade de vida, menores DRT e maior percepção de SO, mas não foi o caso. Além disso, o resultado da relação negativa do SO com a vitalidade e os aspectos sociais pode ser mais profundamente investigado em outros estudos. 


\section{Referências}

Abbad, G. (1999). Um Modelo Integrado de Avaliação de Impacto do Treinamento no Trabalho: IMPACT. 262 f. Tese (Doutorado em Psicologia) - Instituto de Psicologia, Universidade de Brasília, Brasília.

Aquino, A. D., \& Fernandes, A. C. (2013). Qualidade de vida no trabalho. J Health Sci Inst, 31(1), 53-58.

Bizzi, Luciane L. (2013). O bem-estar no trabalho e suas relações com suporte e comprometimento organizacional afetivo: estudo de caso na Universidade Federal De Santa Maria (UFSM). Artigo de Mestrado. Santa Maria, RS, Brasil.

Boehs, S. T. M., Bardagi, M. P., \& Silva, N. (2019). Trabalho, aposentadoria e satisfação de vida em aposentados de uma multinacional. Revista Psicologia, Organizações e Trabalho. 19 (3).

Byrne \& Hochwarter, (2008). Perceived organizational support and performance. Journal of Managerial Psychology. 23 (1), $54-72$.

Borges, R. F. S. (2017). Incentivos, salários e produtividade: Uma análise na perspectiva dos trabalhadores. Tese de Mestrado em Ciências Econômicas e Empresariais. Universidade dos Açores, Ponta Delgada, Portugal.

Borsoi, I.C.F. (2007). Da relação entre trabalho e saúde à relação entre trabalho e saúde mental. Psicol Soc. 19(1), $103-111$.

Brasil. (2003). Instituto Nacional De Seguro Social. Norma técnica sobre distúrbios osteomusculares relacionados ao trabalho - DORT. Instrução Normativa INSS/DC $N^{\circ} 98$ de 05 de dezembro de 2003. https://www.legisweb.com.br/legislacao/?id=75579.

Campos, J. F. (2008). Trabalho em Terapia Intensiva: avaliação dos riscos para a saúde do enfermeiro. Artigo (Mestrado em Enfermagem). Programa de Pós-graduação em Enfermagem. Universidade do Estado do Rio de Janeiro, Rio de Janeiro, Brasil.

Ceribeli, H. B., \& Barbosa, R. M. (2019). Análise da Relação entre Suporte Organizacional Percebido, Exaustão e Comprometimento Organizacional. Reuna, 24(3), 1-19.

Chong, H., White, R. \& Prybutok, V. (2001). Relationship among organizational support, JIT implementation, and performance. Industrial Management + Data Systems. 101(5), 273-280.

Cruz, R. M., \& Maciel, S. K. (2005). Perícia de danos psicológicos em acidentes de trabalho. Estudos e Pesquisas em Psicologia, 5 (2), $120-129$.

Coelho, R. N., Ramalho, G. M., Brito, E. S., Miranda, B. B., Silva, T. I. O. \& Pereira Filho, F. A. A. (2016). A saúde do trabalhador no âmbito universitário: Uma Parceria de Êxito na UFC. Revista de Psicologia, 7, 257-265.

Coelho, G. G., Arruda, A. C. C., Carneiro, A. L. F., Lima, E. S. \& Barros, J. H. O. (2020). O Despertencimento Social de Trabalhadores Terceirizados em uma Universidade Pública: Relato de Experiência. PSI UNISC. 4 (1) 84-92.

Cunha, N. C. R., Oliveira, V. C., Araújo Filho, M. S. de, \& Almeida, R. J. de. (2019). Relação entre o Ambiente Laboral e Problemas Osteomusculares: um estudo com profissionais de Enfermagem em um hospital escola. Revista Brasileira Militar De Ciências, 5(12).

Dabos, G. E., \& Rousseau, D. M. (2004). Mutuality and reciprocity in the psychological contracts of employees and employers. Journal of Applied Psychology. 89(5), 52-72.

Dal Ben, L. W., Carvalho, M. B., Souza, T. M. \& Felli, V. E. A. (2004). A percepção da relação sofrimento/prazer no trabalho de auxiliares de enfermagem e técnicos de enfermagem em internação domiciliária. Revista Cogitare Enfermagem. 9 (2), 73-81.

Dale, A. P. \& Dias, M. D. A. (2018). A 'extravagância' de trabalhar doente: o corpo no trabalho em indivíduos com diagnóstico de LER/DORT. Trab. Educ. Saúde. 16 (1), 263-282.

Dejours, C., Dessors, D. \& Desriaux, F. (1993). Por um trabalho, fator de equilíbrio. Revista de Administração de Empresas, 33 (3), $98-104$.

Faul, F., Erdfelder, E., Lang, A.-G., \& Buchner, A. (May de 2007). G*Power 3: A flexible statistical power analysis program for the social, behavioral, and biomedical sciences. Behavior Research Methods. 39, 175-191.

Ferreira, M.C. \& Mendonça, H. (2012). Saúde e bem-estar no trabalho: dimensões individuais e culturais. Casa do Psicólogo.

Fonseca, K. A. C. (2019). O Clima Organizacional e as doenças ocupacionais de servidores públicos. (Artigo de Mestrado). Instituto Politécnico de Santarém.

Fonseca, E. C., Zeitoune, R. C. G., Sousa, K. H. J. F., Portela, L. F. \& Soares, M. R. C. (2020). Danos à saúde dos trabalhadores de enfermagem de salas de vacinação. Acta paulista de enfermagem. 33. https://doi.org/10.37689/acta-ape/2020AO0147.

Formiga, N. S. \& Souza, M. A. (2014). Comprovação empírica de uma medida psicológica sobre a percepção do suporte organizacional em trabalhadores de diferentes empresas. Boletim - Academia Paulista de Psicologia,34(87), 510-552. http://pepsic.bvsalud.org/scielo.php?script=sci_arttext\&pid=S1415$711 \mathrm{X} 2014000200013 \& \operatorname{lng}=$ pt\&tlng=pt.

Formiga, N. S., Miranda, A. L. B. B., Araújo, I. T. D., Pastana, S. T. G., \& Mafra, A. L. (2018). Evidência da invariância fatorial e validade convergente da escala de suporte organizacional: estudo com trabalhadores brasileiros. Boletim-Academia Paulista de Psicologia, 38(94), 27-35.

Formiga, N. S., Araújo, I. T., Oliveira, B. F., Pastana, S. T., G., Miranda, A., L. B. B., Azevedo, I. M. \& Mafra, A. L. (2019). Suporte organizacional, engajamento e espiritualidade no trabalho: verificação empírica de um modelo teórico concorrente em organizações brasileiras. Boletim - Academia Paulista de Psicologia. 39 (97). 
Formiga, N. S., Freire, B. G. O., Azevedo, I. M. \& Faria, S. S. (2020). Correlatos entre o suporte organizacional, capital psicológico no trabalho e expectativa de futuro: um estudo com trabalhadores brasileiros. Research, Society and Development. 9 (6).

Guedes, M. N. (2008). Terror Psicológico no Trabalho (3a ed.). LTr.

Guimarães, L. L., \& Grubit, S. (2004). Saúde mental e trabalho. Casa do Psicólogo.

Guimarães, José Ribeiro Soares (2012). Perfil do Trabalho Decente no Brasil: um olhar sobre as Unidades da Federação.

Jacques, M. G. C. \& Amazarray, M. R. (2006). Trabalho bancário e saúde mental no paradigma da excelência. Boletim da Saúde. 20 (1), 93-105. http://www.boletimdasaude.rs.gov.br/conteudo/1357/trabalho-bancario-e-saude-mental-no-paradigma-da-exelencia

Laguardia, J., Campos, M. R., Flor, L. S., \& Portugal, F.B. (2015). Questionário genérico de qualidade de vida (SF-36/SF-6D) do Medical Outcomes Study (MOS) (pp. 427-432). In: Clarice Gorenstein; Wang Yuan-Pang; Ines Hungerbühler. (Org). Instrumentos de Avaliação em Saúde Mental. 1ed.Porto Alegre: Artmed Ltda.

Mendes, A. M. B. (2007). Psicodinâmica do trabalho: teoria, método e pesquisa. Casa do Psicólogo.

Mendes, E. V. (Agosto de 2010). As redes de atenção à saúde. Ciência e Saúde Coletiva. 15, 297-305.

Nascimento, R. L. \& Estevam, I. D. (2020). Qualidade de Vida e Saúde Laboral: a Percepção de Servidores Públicos Federais Sobre Um Projeto de Enfrentamento ao Sobrepeso numa Ies Em Natal, RN, Brasil. Novas Edições Acadêmicas.

Ogata, A. \& Simurro, S. (2019). Guia Prático de Qualidade de Vida: como planejar e gerenciar melhor o programa para sua empresa. Editora Alta Books. 2019

OMS. (1998). Promoción de la salud: glosario. Genebra: OMS.

Pafaro, R. C. \& De Martino, M. F. (2004). Estudo do estresse do enfermeiro com dupla jornada de trabalho em um hospital de oncologia pediátrica de Campinas. Rev. Esc. Enferm USP. 38 (2), 152-60.

Paschoal, T. \& Tamayo, A. (2004). Validação da Escala de Estresse no Trabalho. Estudos de Psicologia, 9 (1), 45-52.

Pereira, G. A., Formiga. N. S., \& Estevam, I. D. (2019). Organização, trabalho e saúde: Suporte organizacional, capital psicológico no trabalho e saúde geral em enfermeiros de um hospital público. Montreal: Novas Edições Acadêmicas.

Primo, G. M. G., Pinheiro, T. M. M., \& Sakurai, E. (2007). Absenteísmo no trabalho em saúde: fatores relacionados. Revista Médica de Minas Gerais, 17(1), 294-302.

Ribeiro, L. A \& Santana, L. C, D. (2015). Qualidade de Vida No Trabalho: Fator Decisivo para o Sucesso Organizacional. Revista de Iniciação Científica RIC Cairu. 2 (2),75-96.

Marques, G. S. \& Giongo, C. R. (2016). Trabalhadores bancários em sofrimento: Uma análise da literatura nacional. Revista Psicologia: Organizações e Trabalho, 16(3), 220-235.

Mendes, A. M., \& Morrone, C. F. (2002). Vivências de prazer-sofrimento e saúde psíquica no trabalho: trajetória conceitual e empírica. In A. M. Mendes, L. O. Borges \& M. C. Ferreira (Eds.), Trabalho em transição, saúde em risco. (p. 26-42). Universidade de Brasília.

Miles, J. N. V., \& Shevlin, M. E. (2001). Applying regression and correlation: A guide for students and researchers. Sage Publications.

Mutti, C., Alvim, S., Loiola, E., Gondim, S. M. G., Borges-Andrade, J. (2015). Aprendizagem Experiencial: Articulando Cognições e Emoções para Aprender a Trabalhar. In S. M. G. Gondim \& E. Loiola (Orgs.), Emoções, Aprendizagem e Comportamento Social: Conhecendo para melhor educar em contextos escolares e de trabalho (pp. 53-87). São Paulo, SP: Casa do Psicólogo.

Shantz, A., Arevshatian, L., Alfes, K. \& Bailey, C. (2016). The effect of HRM attributions on emotional exhaustion and the mediating roles of job involvement and work overload. Human Resource Management Journal, 26 (2), 172-191.

Silva, A. K. L., Carabalho, G. P., Jucá, R. M. N. \& Machado, L. S. S. X. (2015). Concepções e expectativas da aposentadoria em trabalhadores da educação pública do Rio Grande do Norte. Aletheia. 47-48, 106-121.

Silva, E. F., Oliveira, K. K., \& Zambroni-de-Souza, P. C. (2011). Saúde mental do trabalhador: o assédio moral praticado contra trabalhadores com LER/DORT. Revista Brasileira Saúde Ocupacional, 36 (123), 56-70.

Siqueira, M. M. M. (1995). Antecedentes do comportamento de cidadania organizacional: a análise de um modelo pós cognitivo. Brasília, 1995. Tese (Doutorado). Universidade de Brasília.

Siqueira, M. M. M. (2005). Esquema mental de reciprocidade e influências sobre afetividade no trabalho. Revista Estudos de Psicologia, 10(1), 83-93.

Siqueira, M. M. M. (2014). Novas medidas do comportamento organizacional: ferramentas de diagnóstico e de gestão. Ar.

Podsakoff, P. M., MacKenzie, S. B., Lee, J. Y., \& Podsakoff, N. P. (2003). Common method biases in behavioral research: a critical review of the literature and recommended remedies. Journal of applied psychology, 88(5), 879-903.

Tabachnick, B.G. \& Fidell, L.S. (2001) Using Multivariate Statistics. (4th ed.), Allyn and Bacon, Boston.

Vasques-Menezes, I. (2012). Saúde do trabalhador: uma breve sistematização. In: Ferreira, M. C.; Mendonça, M. C., H. (Orgs.), Saúde e bem-estar no trabalho: dimensões individuais e culturais. 63-77. Casa do Psicólogo. 
Research, Society and Development, v. 10, n. 8, e36810817380, 2021

(CC BY 4.0) | ISSN 2525-3409 | DOI: http://dx.doi.org/10.33448/rsd-v10i8.17380

Vey, P. Z., Silva, A. C., Braz, M. M. \& Saccol, M. F. (2018). Queixas musculoesqueléticas e qualidade de vida entre bancários de instituições públicas e privadas. Fisioterapia Brasil, 2018;19(6):789-795.

WHOQOL, OMS. (1995). The WHOQOL Group. The World Health Organization quality of life assessment (WHOQOL): position paper from the World Health Organization. Soc Sci Med 1995; 41:1403-9. 